\title{
Proteomic Analysis of Rat Hypothalamus Revealed the Role of Ubiquitin-Proteasome System in the Genesis of DR or DIO
}

\author{
Qi-ming Wang • Hui Yang • De-run Tian • \\ Ying Cai $\cdot$ Zhong-nan Wei $\cdot$ Fei Wang $\cdot$ \\ Albert Ch Yu $\cdot$ Ji-sheng Han
}

Accepted: 8 February 2011 / Published online: 23 February 2011

(c) The Author(s) 2011. This article is published with open access at Springerlink.com

\begin{abstract}
Obesity has become a global epidemic, contributing to the increasing burdens of cardiovascular disease and type 2 diabetes. However, the precise molecular mechanisms of obesity remain poorly elucidated. The hypothalamus plays a major part in regulating energy homeostasis by integrating all kinds of nutritional signals. This study investigated the hypothalamus protein profile in diet-induced obese (DIO) and diet-resistant (DR) rats using two dimensional gel electrophoresis (2-DE) combined with MALDI-TOF/TOF-MS analysis. Twenty-two proteins were identified in the hypothalamus of DIO or DR rats. These include metabolic enzymes, antioxidant proteins, proteasome related proteins, and signaling proteins, some of which are related to AMP-activated protein kinase (AMPK) signaling or mitochondrial respiration. Among these proteins, in comparison with the normal-diet group, Ubiquitin was significantly decreased in DR rats but not changed in DIO rats, while Ubiquitin carboxyl-terminal esterase L1 (UCHL-1) was decreased in DIO rats but not changed in DR rats. The expression level of Ubiquitin and UCHL-1 were further validated using Western blot analysis. Our study reveals that Ubiquitin and UCHL-1 are obesity-related factors in the hypothalamus that may play
\end{abstract}

Qi-ming Wang, Hui Yang are contributed equally to this work.

Q. Wang $\cdot$ H. Yang $\cdot$ D. Tian $(\bowtie) \cdot$ Y. Cai $\cdot$ Z. Wei Department of Anatomy, Tianjin Medical University, 22\# Qixiangtai Road, Tianjin 300070, People's Republic of China e-mail: tiandr@tijmu.edu.cn

F. Wang · A. C. Yu $\cdot$ J. Han

Neuroscience Research Institute, Peking University, 38\#

Xueyuan Road, Beijing 100083, People's Republic of China an important role in the genesis of DR or DIO by interfering with the integrated signaling network that control energy balance and feeding.

Keywords Ubiquitin - UCHL-1 - Diet- induced obesity · Diet-resistant · Two-dimensional gel electrophoresis · Mass spectrometry

\section{Introduction}

Obesity has become a global epidemic, contributing to the increasing burdens of cardiovascular disease, type 2 diabetes, some types of cancer, and premature death $[1,2]$. However, the molecular mechanism in pathogenesis of obesity has not yet been fully elucidated. An increase in food availability, in particular a high-energy diet, seems to account for the development of obesity [3]. Interestingly, a subset of the population appears to be sensitive to dietinduced obesity (DIO), while others are diet-resistant (DR) and can maintain a normal body weight even while on the same diet as DIO individuals [4]. It has been reported that some classic peptides are related to obesity, such as leptin, ghrelin, and some hypothalamic neuropeptides $[3,5,6]$. In our previous study, cocaine and amphetamine- regulated transcript (CART), $\alpha$-melanocyte stimulating hormone $(\alpha-\mathrm{MSH})$ were up-regulated and recognized as anorexigenic neuropeptides to suppress the feeding behavior in DR rats [7].

Recent research confirms that, in the CNS, AMP-activated protein kinase (AMPK) responds to altered AMP/ ATP, nutrients, metabolites, and hormones related to body energy status as well as in peripheral tissue. Hypothalamic AMPK is located in energy-sensing neurons and circuits for body energy homeostasis, and is thus positioned to 
function as a regulator of energy balance [8]. These molecules in AMPK signaling circuits all have important roles in balancing energy intake and expenditure [9]. In addition, many studies show that the Ubiquitin-proteasome system (UPS) plays a crucial role in a wide variety of biological processes, including the cell cycle, cell proliferation, apoptosis and signal transduction [10, 11]. UPS is mainly composed of Ubiquitin, Ubiquitin-activating enzyme, Ubiquitin-conjugating enzymes, Ubiquitin ligases, proteasomes, and deubiquitinating enzymes (Ubiquitincarboxylterminal esterase L1, UCHL1). UCHL-1 appears to be involved in regulating lipid synthesis in adipocyte as well as lipid production by the liver, and could possibly regulate the level of AMPK to influence the development of obesity [10]. Interestingly, UCHL-1 has been found to be up-regulated in the cow hypothalamus in response to food restriction [8]. Ubiquitin involved in the degradation of AMPK by CIDEA (cell death-inducing DNA fragmentation factor-like effector A) gene in adipose tissue [12]. To our knowledge, however, how or whether it is involved in the central regulation of energy balance in DIO and DR rats has never been investigated.

Quite a few previous studies have utilized proteomics to study adipose tissue, pancreas and blood in obesity [1316]. However, no proteomics study on the hypothalamus between DIO and DR rats have been reported. The hypothalamus plays a major part in regulating energy homeostasis by integrating all kinds of nutritional signals. In the present study, two dimensional gel electrophoresis (2-DE) was used in combination with mass spectrometry to search for obesity related factors in the hypothalamus.

\section{Experimental Procedures}

\section{Establishment of DIO and DR Model in Rats}

Three-week-old male (45-55 g) Sprague-Dawley (SD) rats were housed in at $22 \pm 2^{\circ} \mathrm{C}$ on a $12: 12 \mathrm{~h}$ light/dark cycles and fed with standard laboratory chow and water available ad libitum during the first week of the experiment. Animals were randomly divided into two groups as previously described [7]: (1) the control group $(\mathrm{n}=15)$, fed with standard laboratory chow $(3.80 \mathrm{kcal} / \mathrm{g})$; (2) the high-fat $\operatorname{diet}(\mathrm{HIF})$ group $(\mathrm{n}=60)$, fed with high-fat diet containing $4.76 \mathrm{kcal} / \mathrm{g}$. After feeding for 14 weeks, rats in the HIF diet-feeding group that exhibited higher body weights were assigned to the DIO group; their individual body weights surpassed the maximum individual body weights of rats in the control group. Rats of the HIF diet group with lower body weights were assigned to the DR group; their individual body weights were no greater than the average individual body weights of the control group rats.
Samples Preparation for 2-DE and Image Analysis

Under anesthetic with sodium pentobarbital, these rats were sacrificed by decapitation. The brain was dissected from the skull as previously described by Mantzoros CS: a region bordered dorsally by the thalamus, rostrally by the optic chiasm, and caudally by the mamillary bodies was excised [17]. Hypothalamuses removed were immediately frozen in liquid nitrogen. The 2-DE was performed with the Amersham Pharmacia System. A total protein of $1 \mathrm{mg}$ for each sample was applied to an $18 \mathrm{~cm}$ length IPG dry strip ( $\mathrm{pH} \mathrm{3-10} \mathrm{L).} \mathrm{The} \mathrm{IEF} \mathrm{were} \mathrm{performed} \mathrm{on} \mathrm{IPGphor3} \mathrm{IEF}$ system. The IPG strips were equilibrated first for $30 \mathrm{~min}$ in a buffer containing $50 \mathrm{mM}$ Tris- $\mathrm{HCl}, 6.0 \mathrm{M}$ urea, and $0.2 \%$ DTT, followed by $10 \%$ SDS-PAGE on a Protean Plus Dodeca Cell (Bio-Rad, Hercules, USA) After 2-DE, the gels were stained with coomassie brilliant blue R-350. Images were digitized with an ImageScanner (GE Healthcare) and analyzed (spot detection, quantization, gel matching) by using ImageMaster 2D platinum.

In-Gel Digestion and Protein Identification by Mass Spectrometry

Protein spots were excised from the gels for tryptic digestion in-gel. The proteins were then incubated with $10 \mu \mathrm{l} 5 \mu \mathrm{g} / \mathrm{ml}$ trypsin overnight at $37^{\circ} \mathrm{C}$. The peptides were extracted from the gel, mixed, vacuum-dried, and stored for MS analysis. Matrix assisted laser desorption/ ionization time of flight (MALDI-TOF) Tandem mass spectrometry (MALDI-TOF-TOF-MS) were performed using a 4700 Proteomics Analyzer (TOF/TOFTM)(Applied Biosystems, CA, USA). The MS data from both MALDI-MS peptide fingerprint mass spectra and tandem mass spectra in the MS/MS experiments were searched against a subset of rat proteins in the NCBInr protein sequence database, using the Mascot v2.1 search program. Search parameters used were: "trypsin" (enzyme), "carbamidomethyl (C)" (fixed modifications), and "oxidation (M)" (variable modifications).

\section{Western Blot Analysis}

Samples containing $50 \mu \mathrm{g}$ of protein from the hypothalamus of control, DIO, and DR rats were electrophoresed on a $12 \%$ SDS polyacrylamide gel and transferred to a nitrocellulose filter. The membranes were blocked in phosphate-buffered saline for $2 \mathrm{~h}$ and then incubated overnight with an antiUCHL-1 rat polyclonal antibody, an anti-Ubiquitin rat polyclonal antibody, or an anti-actin mouse monoclonal antibody (Abcam, UK). The membranes were then incubated for $1 \mathrm{~h}$ with HRP-conjugated secondary antibody. Specific proteins were detected using an ECL kit (Amersham 
Biosciences, UK). The protein expression level was analyzed using Quantity One Software (Bio-Rad).

\section{Statistics}

Expression levels of the proteins were quantified by analyzing the intensity of each spot. Data were presented as mean \pm SE. Statistical differences between normal and DIO groups and between normal and DR groups were assessed through the one-way analysis of variance (ANOVA), followed by Dunnett's post-hoc test. $P$ values less than $0.05(P<0.05)$ were considered to be statistically significant.

\section{Results}

Effect of HIF Diet on Body Weight and Energy Consumption in DIO and DR Rats

Initial average body weight of the rats in the HIF diet group $(52.7 \pm 0.6 \mathrm{~g})$ did not differ significantly from that of the rats in the control group $(52.2 \pm 1.3 \mathrm{~g})(P>0.05)$. At 14-weeks, the average body weight of rats in the HIF diet group (including DIO and DR groups) was $631.2 \pm 22.6 \mathrm{~g}$, which was $13.9 \%$ more than that of rats in the control group $(P<0.01)$. In the HIF diet group, the average body weight of DIO rats was $688.9 \pm 28.3 \mathrm{~g}$, which was $29.6 \%$ more than that of the DR rats $(P<0.01)$. At $12-14$ weeks, a significantly higher energy intake per day was observed in DIO group (138.6 $\pm 12.9 \mathrm{kcal} /$ day) as compared to the DR group $(112.3 \pm 10.2 \mathrm{kcal} /$ day $)$ and the control group $(110.2 \pm 13.0 \mathrm{kcal} /$ day $)(P<0.001)$ (Table 1$)$.

Comparative Expressed Profiles of 2-DE Analysis and Identified Proteins Differentially Expressed in DR and DIO Groups

To detect proteins differently expressed in the hypothalamus, we investigated the hypothalamus protein profile in normal, DIO and DR rats by 2-DE. The 2-DE protein patterns showed a resolution of approximately 1,280 \pm 15 , $1,250 \pm 10$, and 1,260 \pm 12 protein spots in normal, DIO,

Table 1 Body weight and energy intake of rats in control, DR and DIO groups (Mean $\pm \mathrm{SE}$ )

\begin{tabular}{llll}
\hline Groups & $\begin{array}{l}\text { Numbers } \\
\text { of rats }\end{array}$ & $\begin{array}{l}\text { Averaged body } \\
\text { weights }(\mathrm{g})\end{array}$ & $\begin{array}{l}\text { Energy intake } \\
(\mathrm{kcal} / \mathrm{d})\end{array}$ \\
\hline Control & 15 & $554.2 \pm 20.2$ & $110.2 \pm 13.0$ \\
DR & 15 & $531.4 \pm 24.8$ & $112.3 \pm 10.2$ \\
DIO & 30 & $688.9 \pm 28.3 * * *$ & $138.6 \pm 12.9 * * *$ \\
\hline
\end{tabular}

$* * * P<0.001$ compared with control group or DR group and DR groups, respectively. Protein spot positions and intensities were compared between gel patterns of normal and DIO groups and between that of normal and DR groups. By comparing 2-DE gel profiles from normal and DIO groups and from normal and DR groups, respectively (Fig. 1), we identified 22 differentially expressed protein spots in two groups by PMF or MS/MS analysis. Among them are 7 up-regulated and 5 down-regulated proteins in DR groups and 7 up-regulated and 7 down-regulated proteins in DIO groups in comparison to the normal group, respectively. Four down-regulated proteins are same in both DIO and DR groups. These proteins are further categorized into 7 metabolic enzymes (spot 1,2,5,8,13,16,18 Table 2), 4 signaling proteins (spot 6, 7, 17, 20), 2 proteasome related proteins (spot 10 and 12), 1 modulin of cell cycle (spot 19), 1 antioxidant protein (spot 3), 1 specific protein of neurocyte (spot 21), 3 proteins with other functions (spot 4, 15 and 22), and 3 unknown proteins (spot 9, 11 and 14). Arrows in Fig. 1a, b, c indicated the variant spots, which showed a significant difference between two gel patterns. Table 2 displays the identification of proteins differentially expressed in DIO or DR group in comparison to normal group.

Differential Expressions of Ubiquitin Carboxyl-

Terminal Hydrolase L1 (UCHL-1), Ubiquitin in DIO or DR Group Compared With Control Group

To probe the expression of UCHL-1 and Ubiquitin in obesity, we cut out spot 10 and spot 12 (marked with arrows) from the gel and verify their identities by MALDITOF-MS or MS/MS. The trypsin peptide spectrums of spot 10 and spot 12 are shown in Figs. 2 and 3. Several peaks were found in the theoretic sequence of UCHL-1 with a score of 136 , covering $72 \%$ of the whole protein, and in that of Ubiquitin with a score of 167 , covering $81 \%$ of the whole protein.

To further characterize the changes in UCHL-1 and Ubiquitin in DIO and DR groups, we quantified UCHL-1 and Ubiquitin levels using Western blotting. In agreement with previous data from MS or MS/MS, the level of UCHL-1 expression in the DIO group was significantly lower than that in the control $(P<0.05)$. Similarly, the level of Ubiquitin expression in the DR group was significantly lower than that in the control group $(P<0.05)$ (Fig. 4).

\section{Discussion}

Obesity, one of the most prevalent diseases in the world, is associated with the consumption of a high-energy diet [18]. The task of identifying causative or contributory factors is 
Fig. 1 Proteome maps of the hypothalamus of rats in normal (a), DIO (b), and DR(c) groups. Proteins were separated by electrophoresis on IPG gels (pI 3-10) and by SDS-PAGE, stained with coomassie brilliant blue R-350. Identified protein spots are marked by arrows with numbers
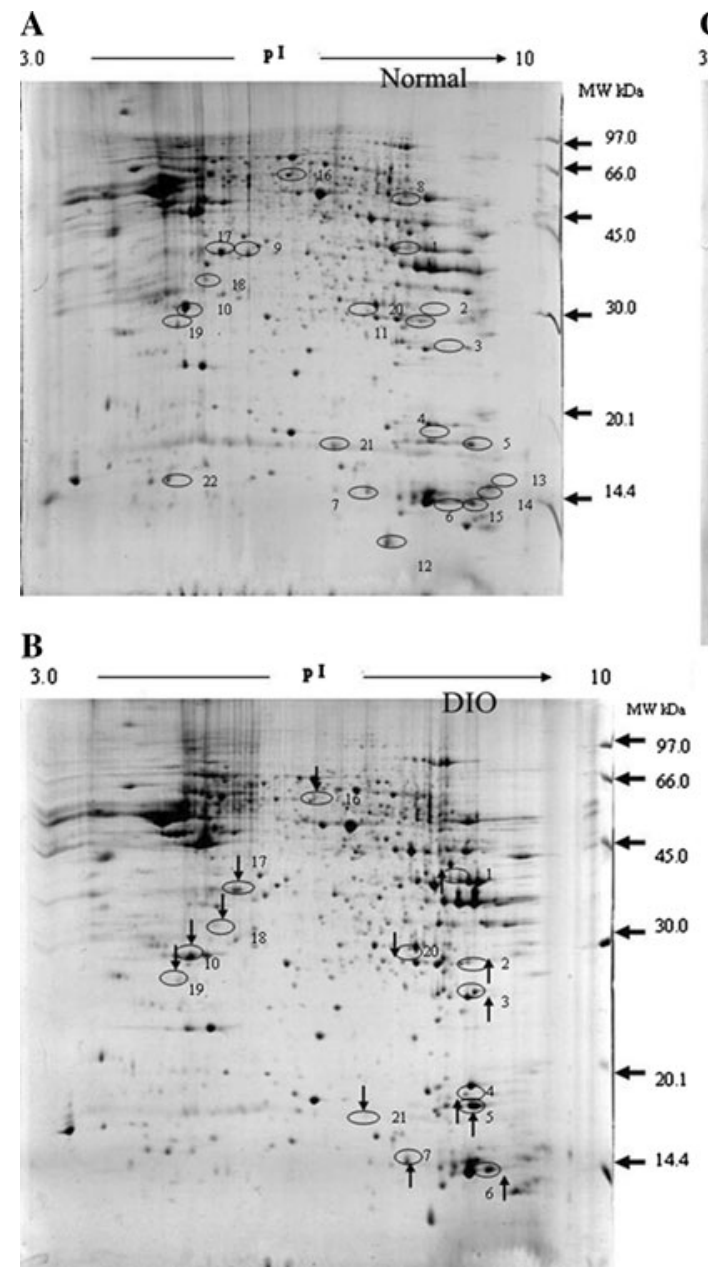

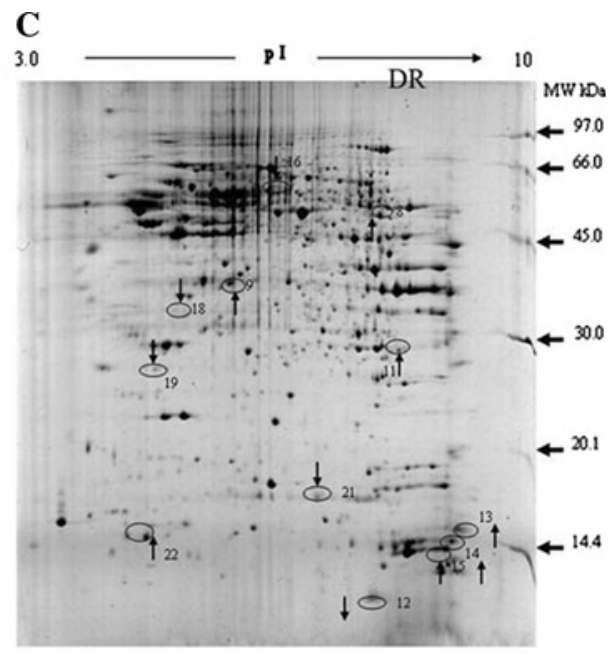

difficult because of the multi-etiologic nature of obesity and the multiple metabolic perturbations that occur in obese individuals. DIO and DR rat models have been used in studying the role of nervous system in regulating feeding behavior and energy homeostasis [19].

The hypothalamus has long been recognized as a major site in the central nervous system to integrate a spectrum of internal and external environmental information for energy homeostasis [20]. In the present study, some identified proteins are involved in energy metabolism, UPS, and oxidative stress. Ubiquitin and UCHL-1 are both components of UPS. Ubiquitin is recycled by proteolytic removal from its conjugating protein by deubiquitinating enzymes (DUBs). Our results showed that, compared to the normal control group, Ubiquitin was down-regulated in the DR group, though there was no significant change in the DIO group. Interestingly, one key member of deubiquitinating enzymes, UCHL-1, was down-regulated only in the DIO group, but not in the DR group. Ubiquitin carboxy terminal hydrolase (UCH) is a subclass of DUBs that catalyzes the hydrolysis of $\mathrm{COOH}$-terminal ubiquityl esters and amides. It removes Ubiquitin from ubiquitinated cellular proteins, thereby preventing them from targeted degradation via the proteasome pathway. In the DIO group, UCHL-1 with a lower expression level could destroy the initial balance between ubiquitin monomers and ubiquitin conjugates [21]. The decrease of UCHL-1 in DIO rats may cause the abnormal regulation of $\mathrm{Ub}$ system, as shown in $\mathrm{Ubb}^{-/-}$ mice[22].The depletion of Ub from hypothalamic neurons may interfere with the integrated signaling network that controls energy balance and feeding in complex ways.The obesity in DIO rats is likely the result of impaired function of multiple hypothalamic networks such as melanocortin-3 receptor(Mc3r) signaling or AMPK signaling pathways [23-25]. By contrast, we think the decrease of Ub in DR rats might be associated with the normal facilitation of $\mathrm{Ub}$ system. The hypothesis needs to be confirmed further.

In the DR group, protein disulfide isomerase associated 3(PDIA3)(spot16) was also down-regulated in addition to Ubiquitin. The protein disulphide-isomerase (PDI) family encompasses several highly divergent proteins that are involved in the processing and maturation of secretory proteins in the endoplasmic reticulum (ER). Some studies had demonstrated that PDI can interact with Ubiquitin, 
Table 2 Identification of proteins differentially expressed in hypothalamus of DIO or DR in comparison to normal control group

\begin{tabular}{|c|c|c|c|c|c|c|c|}
\hline Group & Spot No* & Spot ID & MW & $\mathrm{pI}$ & Coverage $\%$ & Fold change & Protein name \\
\hline \multirow[t]{14}{*}{ DIO } & 1 & gil202837 & 39235 & 8.32423 & 36 & 1.62 & Aldolase A \\
\hline & 2 & gil8393502 & 25887 & 8.52664 & 32 & 2.31 & Glutathione S-transferase,m1 \\
\hline & 3 & gil16923958 & 26011 & 8.26652 & 41 & 2.54 & Peroxiredoxin 1 \\
\hline & 4 & gil75991707 & 18629 & 8.19503 & 52 & 1.87 & Destrin \\
\hline & 5 & gil8394009 & 17863 & 8.68517 & 50 & 1.72 & Peptidylprolyl isomerase A \\
\hline & 6 & gil6981010 & 15319 & 7.39654 & 33 & 2.06 & Similar to zinc finger protein \\
\hline & 7 & gil149060386 & 18652 & 7.08953 & 86 & 2.22 & Fibroblast growth factor receptor 2 \\
\hline & 10 & gil61098212 & 24822 & 5.14365 & 72 & 0.38 & Ubiquitin carboxyl-terminal hydrolase L1 \\
\hline & 16 & gil8393322 & 56526 & 5.80976 & 37 & 0.51 & Protein disulfide isomerase associated 3 \\
\hline & 17 & gil984553 & 37369 & 5.39329 & 22 & 0.52 & G protein beta 1 subunit \\
\hline & 18 & gil85541051 & 33094 & 5.44817 & 48 & 0.44 & Pyridoxal phosphate phosphatase \\
\hline & 19 & gil13242243 & 38177 & 7.81951 & 12 & 0.45 & Axin2 \\
\hline & 20 & gil149050967 & 15313 & 7.35061 & 31 & 0.48 & E2F transcription factor 6 \\
\hline & 21 & gil4454311 & 14184 & 8.89452 & 32 & 0.49 & Myelin basic protein \\
\hline \multirow[t]{12}{*}{ DR } & 8 & gil189011657 & 56418 & 8.45528 & 15 & 1.56 & 4-aminobutyrate aminotransferase \\
\hline & 9 & gil55825 & 45637 & 6.28296 & 29 & 2.01 & rCG25241, isoform CRA_b \\
\hline & 11 & gil51948422 & 28155 & 9.01211 & 40 & 1.63 & es1 protein \\
\hline & 12 & gil51701919 & 8520 & 7.86224 & 81 & 0.42 & Ubiquitin \\
\hline & 13 & gil189011657 & 13550 & 9.302145 & 45 & 2.78 & Ubiquinol-cytochrome $\mathrm{c}$ reductase binding protein \\
\hline & 14 & gil54824 & 15982 & 9.02548 & 64 & 3.15 & Unnamed protein product \\
\hline & 15 & gil60678292 & 15275 & 8.45 & 37 & 2.44 & Hemoglobin alpha 2 chain \\
\hline & 16 & gil8393322 & 56526 & 5.80976 & 22 & 0.50 & Protein disulfide isomerase associated 3 \\
\hline & 18 & gil85541051 & 33094 & 5.44817 & 48 & 0.48 & Pyridoxal phosphate phosphatase \\
\hline & 19 & gil13242243 & 38177 & 7.81951 & 12 & 0.49 & Axin2 \\
\hline & 21 & gil4454311 & 14184 & 8.89452 & 32 & 0.51 & Myelin basic protein \\
\hline & 22 & gil157820637 & 18171 & 5.51243 & 17 & 2.05 & Lymphatic vessel endothelial hyaluronan receptor \\
\hline
\end{tabular}

All the proteins were identified with a MALDI-TOF-MS or MS/MS, and the quality of match was evaluated at the significant level $(P<0.05)$ for peptide fingerprint mass spectra and/or peptide sequencing spectra

* The spot number is according to spot position in 2-DE. Spot ID is the accession number from NCBInr $M W$ molecular weight (Da), $p I$ isoelectric point

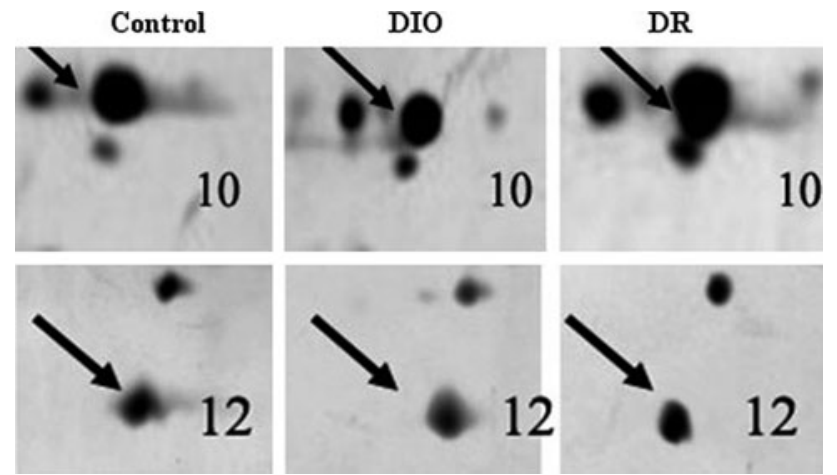

Fig. 2 The panel shows enlarged spot 10 and spot 12 of the 2DE protein profile representing changes in control, DIO and DR groups

which has a ubiquitin-like domain and a ubiquitin-associated domain. Ubiquitin could serve as an adaptor protein that both interacts with PDI and mediates the delivery of poly-ubiquitylated proteins to the proteasome in the vicinity of the ER membrane [26]. Down-regulation of PDIA3 in the DR group might delay the hydrolysis of Ubiquitin by proteasome and compensate the role of ubiquitin-mediated AMPK degradation in DR rats. Kuhla's [8] research confirmed that the UPS activated with upregulation of UCHL-1 in the hypothalamus of cows after energy restriction. UCHL-1, a neuron specific peptidase, could eliminate the misfolded or post-translationally modified proteins by energy deprivation. Our results also displayed abnormal activation of UPS in the hypothalamus of rats fed with an HIF diet. These findings suggest that UPS might play a role in the pathogenesis of DIO and DR. The disruption of $\mathrm{Ub}$ pool dynamics could be a common neuropathogenic mechanism in most late-stage neurodegeneratively diseases $[22,27]$. However, the exact mechanism needs to be confirmed further. In addition to, some 


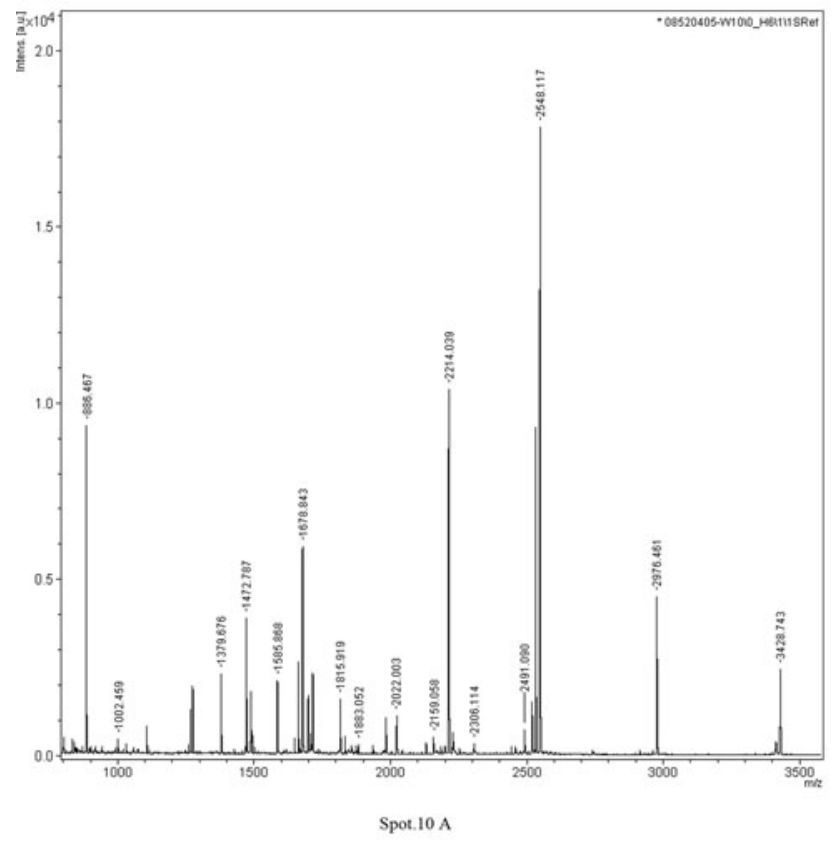

Sequence Coverage: $\mathbf{7 2} \%$

Matehed peptides shown in Bold and Italic

1 MQLKPMEINP EMLNKVLAKL GVAGQWRFAD VLGLEEETLG

SVPSPACALL

51 LLFPLTAQHE NFRKKQIEEL KGQEISPKVY FMKQTIGNSC

GTIGLIHAVA

101 NNQDKLEFED GSVZKQFLSE TEKLSPEDRA KCFEKNEAIQ

AAHDSVAQEG

151 QCRIDDKVNF HFILFNNVDG HLYELDGRMP FPINHGASSE DSLLQDAAKV

201 CREFTEREQG EIRFSAVALC KAA

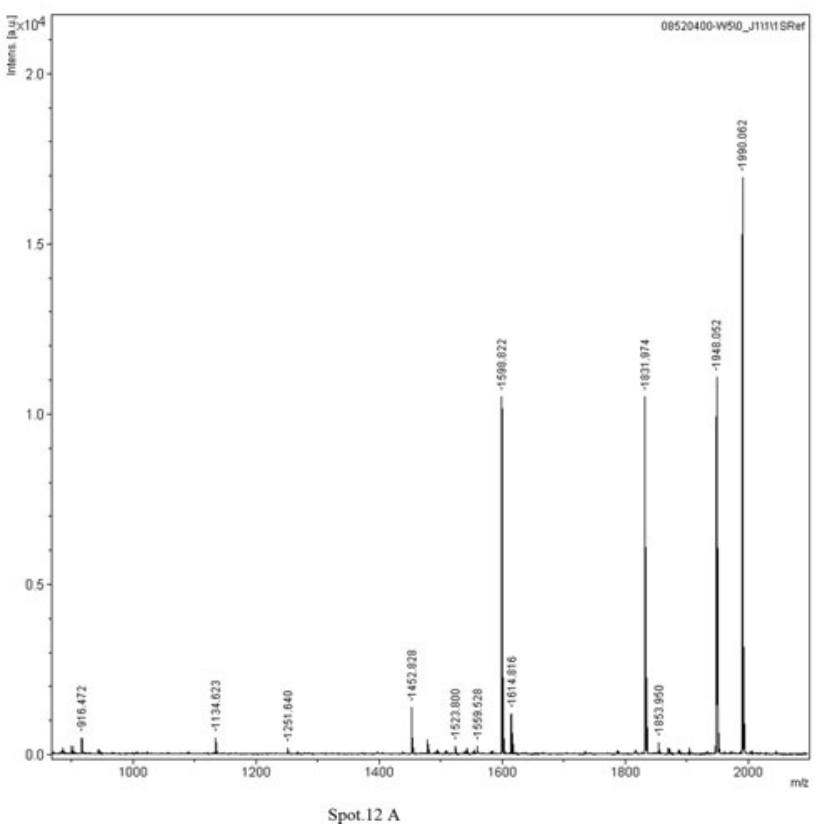

Sequence Coverage: $\mathbf{8 1} \%$

Matched peptides shown in Bold and Italic

1 MQIFVKTLTG KTITLEVEPS DTIENVKAKI QDKEGIPPDQ

QRLIFAGKQL

51 EDGRTLSDYN IQKESTLHLVLRLRGG

Spot.12 B

Fig. 3 The analysis of spot 10 and spot 12 using MALDI-TOF-MS. a Peptide mass fingerprinting, b The peptide coverage maps (matched peptides between spot 10 and UCHL-1, spot 12 and Ubiquitin)

of differential expression proteins such as pyridoxal phosphate phosphatase (spot18) related to AMPK signaling by acetyl-CoA carboxylase(ACC), peroxiredoxin 1 (spot3) and glutathione S-transferase, m1 (spot2) related to mitochondrial respiration with uncoupling protein 2(UCP2). Whether these proteins act as food intake regulatory factors linked to genesis of DIO and/or DR in hypothalamus metabolism remains to be identified.
In summary, the proteomic analysis identifies potential new candidate molecules involved in maintaining energy homeostasis in the hypothalamus of DIO or DR rats. Our study reveals that Ubiquitin and UCHL-1 are obesityrelated factors in the hypothalamus and they may play an important role in the genesis of DR or DIO by interfering with the integrated signaling network that control energy balance and feeding. 
Fig. 4 Expression of the UCHL-1 and Ubiquitin in control, DIO and DR groups. Protein expressions were analyzed via Western blot. Compared to the control group, expression of UCHL-1 was significantly decreased in the DIO group $(P<0.05)(\mathbf{a}, \mathbf{c})$ and expression of Ubiquitin was significantly decreased in the DR group $(P<0.05)(\mathbf{b}, \mathbf{d})$. The densities of the bands of UCHL-1 and Ubiquitin were normalized against actin
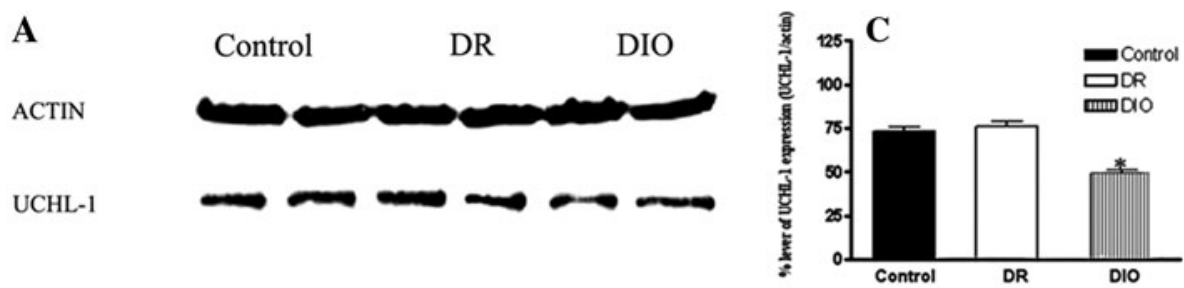

B

Control

DR

DIO

ACTIN

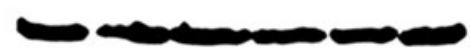

UBIQUITIN

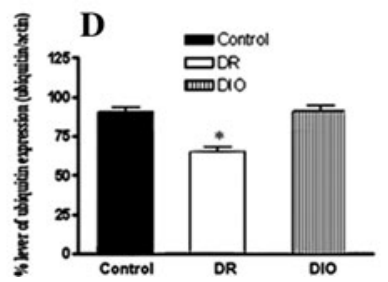

Acknowledgments We thank Dr Wang Hong-li from National Center of Biomedical Analysis, China for technical support. This study was supported by the National Natural Science Foundation of China (No. 3087791) and Natural Science Foundation of Tianjin (07JCDJC08100).

Open Access This article is distributed under the terms of the Creative Commons Attribution Noncommercial License which permits any noncommercial use, distribution, and reproduction in any medium, provided the original author(s) and source are credited.

\section{References}

1. Yang W, Kelly T, He J (2007) Genetic epidemiology of obesity. Epidemiol Rev 29:49-61

2. Novak CM, Kotz CM, Levine JA (2006) Central orexin sensitivity, physical activity, and obesity in diet-induced obese and dietresistant rats. Am J Physiol Endocrinol Metab 290:E396-E403

3. Yuan Q, Fontenele-Neto, JD Fricker LD (2004) Effect of voluntary exercise on genetically obese Cpefat/fat mice: quantitative proteomics of serum. Obes Res 12:1179-1188

4. Levin BE, Triscari J, Hogan S, Sullivan AC et al. (1987). Resistance to diet-induced obesity: food intake, pancreatic sympathetic tone, and insulin. Am J Physiol 252:R471-R478

5. Huang XF, Han M, Storlien LH (2003) The level of NPY receptor mRNA expression in diet-induced obese and resistant mice. Brain Res Mol Brain Res 115:21-28

6. Huang XF, Yu Y, Li Y et al (2008) Ventromedial hypothalamic NPY Y2 receptor in the maintenance of body weight in dietinduced obesity in mice. Neurochem Res 33:1881-1888

7. Tian DR, Li XD, Shi YS et al (2004) Changes of hypothalamic alpha-MSH and CART peptide expression in diet-induced obese rats. Peptides 25:2147-2153

8. Kuhla B, Kuhla S, Rudolph PE et al (2007) Proteomics analysis of hypothalamic response to energy restriction in dairy cows. Proteomics 7:3602-3617

9. Xue B, Kahn BB (2006) AMPK integrates nutrient and hormonal signals to regulate food intake and energy balance through effects in the hypothalamus and peripheral tissues. J Physiol 574:73-83

10. Wing SS (2008) The UPS in diabetes and obesity. BMC Biochem 9:1-6

11. Wang WJ, Li QQ, Xu JD et al (2008) Over -expression of Ubiquitincarboxy terminal hydrolase-L1 induces apoptosis in breast cancer cells. Int J Oncol 33:1037-1045
12. Ronnett GV, Ramamurthy S, Kleman AM et al. (2009) AMPK in the brain: its roles in energy balance and neuroprotection. J Neurochem 109(Suppl 1):17-23

13. Hess S, Chen X (2008) Applications of proteomics to the study of adipose tissue. Methods Mol Biol 456:131-140

14. Dayarathna MK, Hancock WS, Hincapie M (2008) A two step fractionation approach for plasma proteomics using immunodepletion of abundant proteins and multi-lectin affinity chromatography: application to the analysis of obesity, diabetes, and hypertension diseases. J Sep Sci 31:1156-1166

15. Schmid GM, Converset V, Walter N et al (2004) Effect of high-fat diet on the expression of proteins in muscle, adipose tissues, and liver of C57BL/6 mice. Proteomics 4: 2270-2282

16. Berkane AA, Nguyen HT, Tranchida F et al (2007) Proteomic of lipid rafts in the exocrine pancreas from diet-induced obese rats. Biochem Biophys Res Commun 355:813-819

17. Ziotopoulou M, Mantzoros CS, Hileman SM et al (2000) Differential expression of hypothalamic neuropeptides in the early phase of diet-induced obesity in mice. Am J Physiol Endocrinol Metab 279:E838-E845

18. Schrauwen P, Westerterp KR (2000) The role of high-fat diets and physical activity in the regulation of body weight. Br J Nutr $84: 417-427$

19. Mercer JG, Speakman JR (2001) Hypothalamic neuropeptide mechanisms for regulating energy balance: from rodent models to human obesity. Neurosci Biobehav Rev 25:101-106

20. Tian DR, Li XD, Wang F et al (2005) Up-regulation of the expression of cocaine and amphetamine-regulated transcript peptide by electroacupuncture in the arcuate nucleus of dietinduced obese rats. Neurosci Lett 383:17-21

21. Sarkar P, Sarkar S, Ramesh V et al (2008) Proteomic analysis of mouse hypothalamus under simulated microgravity. Neurochem Res 3:2335-2341

22. Ryu KY, Garza JC, Lu XY et al (2008) Hypothalamic neurodegeneration and adult-onset obesity in mice lacking the Ubb poly Ubiquitin gene. Proc Natl Acad Sci USA 105:40164021

23. Mountjoy PD, Bailey SJ, Rutter GA (2007) Inhibition by glucose or leptin of hypothalamic neurons expressing neuropeptide $\mathrm{Y}$ requires changes in AMP- activtaed protein kinase activity. Diabetologia 50:168-177

24. Chau-Van C, Gamba M, Salvi R (2007) Metformin inhibits adenosine 5-mono- phosphate activated kinase activation and prevents increases in neuropeptide $\mathrm{Y}$ expression in cultured hypothalamic neurons. Endocrinology 148:507-511 
25. Qi J, Gong J, Zhao T et al (2008) Downregulation of AMPactivated protein kinase by Cidea-mediated ubiquitination and degradation in brown adipose tissue. EMBO J 27:1537-1548

26. Ko HS, Uehara T, Nomura Y (2002) Role of ubiquilin associated with protein- disulfide isomerase in the endoplasmic reticulum in stress-induced apoptotic cell death. J Biol Chem 277:3538635392
27. Lowe J, Blanchard A, Morrell K (1988) Ubiquitin is a common factor in intermediate filament inclusion bodies of diverse type in man, including those of Parkinson's disease, Pick's disease, and Alzheimer's disease, as well as Rosenthal fibres in cerebellar astrocytomas, cytoplasmic bodies in muscle, and mallory bodies in alcoholic liver disease. J Pathal 155:90-94 